Jordi Rello

Jeffrey Lipman

\section{Antibiotic prescription for respiratory tract infections in ventilated patients: where are we heading?}

Received: 23 April 2013

Accepted: 25 May 2013

Published online: 28 June 2013

(C) Springer-Verlag Berlin Heidelberg and ESICM 2013

This editorial refers to the article available at doi:10.1007/s00134-013-2997-6.

\section{J. Rello (河)}

Critical Care Department, Hospital Universitari Vall d'Hebron, CIBERES, Universitat Autonoma de Barcelona, Barcelona, Spain e-mail: jrello@crips.es

\section{J. Lipman}

Department of Intensive Care Medicine, Burns Trauma Critical Care Research Centre, Royal Brisbane and Womens Hospital, The University of Queensland, Brisbane, Australia

Respiratory infections in intubated patients are the main cause of antibiotic prescription in medical ICU patients. Particularly frequent in multiple trauma patients, they are relatively uncommon in the postoperative period of cardiac surgery, focusing on the minority of patients that require $>24 \mathrm{~h}$ of mechanical ventilation, mainly associated with surgical complications such as low cardiac output or neurological injury. Lacking a gold standard for diagnosis, with low specificity of chest X-rays, it is a common practice to prescribe antibiotics to patients with purulent respiratory secretions. Optimal duration of therapy is unknown, but standard of care in a large multicentre study in 27 ICUs from nine countries in Europe [1] was 8 days of antibiotic prescription. This study detailed the drivers of antibiotic prescription for intubated patients in Europe, including causes of antiMRSA prescription. Further research is needed to assess newer strategies of therapy and the impact on emerging resistance. Antibiotic stewardship practices are recommended in ICU care, but it remains unclear what practices are associated with benefits. Indeed, the real impact on emergence of resistance and outcomes is largely unknown.

We commend Bouza et al. [2] for addressing a perplexing intensive care problem, that of ventilatorassociated pneumonia (VAP). They randomised postoperative cardiac surgical patients to either 3 days of prophylactic antibiotics (meropenem and linezolid) or "standard of care". Whilst they could not demonstrate patient-centred outcome benefits (i.e. mortality, ventilator days, ICU length of stay), they purport to show fewer infections in these patients within the ICU.

We suggest there are other ways to address this enigma. Let us look a little into the future of the intensive care treatment of patients who have been ventilated for a number of days and who develop a respiratory complication assumed to be an infection.

One of the eternal problems in ICU is actually differentiating the inflammatory response from an infective response. This often means trying to determine if bacteria are merely colonising the patient or whether they are actually causing disease. Herein lies the first problem, how do we actually know that the bacterial growth we get from the respiratory tract is definitively causing the inflammatory response of the patient who is being ventilated? How do we know what we grow from the upper respiratory tract is causing disease in the lower tract?

Biomarkers, required for pre-emptive therapy, are not often good enough to help us in this dilemma [3], and whilst PCT may be currently the forerunner in this respect $[4,5]$, there is an ongoing search for one with better sensitivity and specificity $[5,6]$. It may be that genomics will provide a better answer for us [6,7], although there still needs to be more research on any gene expression before such a marker (or markers) have widespread clinical applicability. 
Another area of intense research is to identify causative organisms more timeously [4, 8, 9]. One of the most promising techniques is matrix-assisted laser desorption ionization time-of-flight mass spectrometry or MALDITOF MS (sometimes without the MS). This technology allows identification of both Gram-positive and Gramnegative bacteria (to a species level) within a few minutes, with a relatively low bacterial load being necessary for identification. A recent large observational study has demonstrated clinical benefit for this quick turn-around time [9]. In fact, we would even suggest that to have such a system, to accurately give us which organism is our pathogen and its sensitivity as a point-of-care test, is an obvious holy grail for intensivists. We believe this is not too far into the future.

The next issue we believe to be a problem is the sensitivity and specificity of the current diagnostic criteria for VAP [10]. The definitions include many subjective components (chest radiography, respiratory secretion assessment and even chest auscultation), and hence the inter-observer variability for detecting VAP is large. Klompas et al. [11] proposed a simple, objective surveillance definition for ventilator-associated complications (VAC), shifting the focus of surveillance from pneumonia alone to complications. Some of these events may be unavoidable consequences of caring for the critically ill patients, so zero may no longer be a realistic goal. There is now at least one study showing better discriminatory powers of VAC as against VAP [12], with more studies on the way. Basically, VAC has objectively defined parameters that allow more widespread detection of pulmonary complications in ventilated patients. VAC has simplicity and objectivity and is more consistently associated with adverse outcomes than VAP.

Lastly, in relation to the future, we believe inhaled antibiotics will become standard of care. Interestingly, recent data suggest that outcomes in patients with multidrug-resistant non-fermentative GNB are comparable when high dose colistin $(5 \mathrm{MU} / 8 \mathrm{~h})$ is nebulised, either alone or in combination with parenteral therapy [13]. This is a promising way to administer high concentrations in the distal airways, facilitating bacterial killing in the presence of organisms with high MICs. Complications, such as blocking of the expiratory limb of the ventilator or bronchospasm, are limitations that require further research.

Getting back to the current study by Bouza et al. [2], their findings add information that 3 days of therapy is associated with increased resistance to linezolid. We know that more than 2 days of antimicrobial agents can be associated with increased resistance [14], modifying gut flora. Recent information suggests that prevention of VAP can be achieved with one single dose, such as in surgical prophylaxis, adding evidence for appropriate stewardship in the ICU [15]. The fundamental issue with Bouza et al.'s study is whether 3 days of antibiotics causes any "collateral damage" [2]. On the top of this, even they have shown some downside of their 3-day regimen - namely development of linezolid-resistant organisms.

In summary, the study by Bouza et al. [2] is an additional piece in the puzzle of stewardship, adding evidence on the risk of "collateral damage" of 3 days of meropenem plus linezolid. The breakpoints for colonization, bronchitis or pneumonia are unclear, and it is more important to focus on the need of vasopressors and complications such as the impact on oxygenation. Therefore, we believe that patients with ventilator-associated respiratory infections have to be viewed as severe sepsis or septic shock in intubated patients, the relevant and common presentations at the bedside. Further studies have to focus on outcome parameters of VAC, and a new paradigm of management is required.

Conflicts of interest J. R. served in speakers bureau or/and advisory boards for Pfizer, ROCHE, KENTA, Astra-Zeneca, Merck and Astellas.

\section{References}

1. Rello J, Ulldemolins M, Lisboa T, Koulentis D, Mañez R, Martin-Loeches I, De Waale J, Putensen C, Guven M, deja M, Diaz E, EUVAP/CAP Study group (2011) Determinants of prescription and choice of therapy in hospital-acquired pneumonia and ventilator-associated pneumonia. Eur Resp J 37:1332-1339

2. Bouza E, Perez-Granda MJ, Hortal J, Barrio JM, Cercenado E, Munoz P (2013) Pre-emptive broad-spectrum treatment for ventilator-associated pneumonia in high-risk patients. Intensive Care Med. doi: 10.1007/s00134-013-2997-6
3. Calfee CS, Pugin J (2012) The search for diagnostic markers in sepsis: many miles yet to go. Am J Respir Crit Care Med 186:2-4

4. Póvoa P, Salluh JI (2012) Biomarkerguided antibiotic therapy in adult critically ill patients: a critical review. Ann Intensive Care 2:32

5. Mitsuma SF, Mansour MK, Dekker JP (2013) Promising new assays and technologies for the diagnosis and management of infectious diseases. Clin Infect Dis 56:996-1002
6. Sutherland A, Thomas M, Brandon R, Brandon R, Lipman J, Tang B, McLean A, Pascoe R, Price G, Nguyen T, Stone G, Venter D (2011) Development and validation of a novel molecular biomarker diagnostic test for the early detection of sepsis. Crit Care 15:R149

7. Johnson SB, Lissauer M, Bochicchio GV, Moore R, Cross AS, Scalea TM (2007) Gene expression profiles differentiate between sterile SIRS and early sepsis. Ann Surg 245:611-621 
8. Prod'hom G, Bizzini A, Durussel C, Bille J, Greub G (2010) Matrix-assisted laser desorption ionization-time of flight mass spectrometry for direct bacterial identification from positive blood culture pellets. J Clin Microbiol 48:1481-1483

9. Clerc O, Prod'hom G, Vogne C, Bizzini A, Calandra T, Greub G (2013) Impact of matrix-assisted laser desorption ionization time-of-flight mass spectrometry on the clinical management of patients with gramnegative bacteremia: a prospective observational study. Clin Infect Dis 56:1101-1107
10. Klompas M (2012) What can we learn from international ventilator-associated pneumonia rates? Crit Care Med 40:3303-3304

11. Klompas M (2013) Complications of mechanical ventilation. The CDC's new surveillance paradigm. N Engl J Med 368:1472-1475

12. Hayashi Y, Morisawa K, Klompas M, Jones M, Bandeshe H, Boots R, Lipman J, Paterson DL (2013) Towards improved surveillance: the impact of ventilator-associated complications (VAC) on length of stay and antibiotic use in patients in intensive care units. Clin Infect Dis 56:471-477
13. Lu Q, Luo R, Yang J, Zahr N, Aubry A, Golmard JL, Rouby JJ (2012) Efficacy of high-dose nebulized colistine in ventilator-associated pneumonia caused by multidrug resistant Pseudomonas aeruginosa and Acinetobacter baumannii. Anesthesiology 117:135-147

14. Rello J, Ausina V, Ricart M, Castella J, Prats G (1993) Impact of prior antibiotic therapy on etiology and outcomes of ventilator-associated pneumonia. Chest 104:1230-1235

15. Rello J (2013) Antibiotic stewardship in the ICU. Chest 143:1195-1196 